\title{
Los diseños de método mixto en la investigación en educación: Una experiencia concreta
}

\section{Mixed Method Designs in Education Research: a Particular Experience}

\author{
Zulay Pereira Pérez ${ }^{1}$ \\ División de Educación Básica \\ Centro de Investigación y Docencia en Educación \\ Universidad Nacional \\ Heredia, Costa Rica \\ zulaypereira@yahoo.com
}

Recibido 14 de octubre de 2010 • Aceptado 09 de marzo de 2011

\begin{abstract}
Resumen. La utilización de los diseños de método mixto se constituyen, día a día, en una excelente alternativa para abordar temáticas de investigación en el campo educativo. Este ensayo plantea una revisión acerca de dichos diseños y los ejemplifica con una experiencia concreta de investigación realizada por la propia autora, en la que fue aplicado un diseño de método mixto para una temática en el campo educativo.
\end{abstract}

Palabras claves. Educación, diseños, método mixto, investigación.

Abstract. Mixed method designs are becoming an excellent option to approach research topics in the education field. This essay proposes a review of such designs and illustrates their application through a particular research experience, in which a mixed method design was applied by the author to a specific topic in the education field.

Keyword. Education, designs, mixed methods, research.

\section{Introducción}

Investigar es un proceso inherente a la vida misma; no obstante, la investigación en educación conlleva, además de un interés y una necesidad, una búsqueda para la profundización y comprensión de los fenómenos educativos, más allá de lo meramente evidente. Involucra una búsqueda

\footnotetext{
Doctora en Educación, de la Universidad Estatal a Distancia, Costa Rica. Catedrática de la Universidad Nacional, Costa Rica. Máster y Licenciada en Psicología de la Universidad de Costa Rica. Adscrita al Colegio Profesional de Psicólogos de Costa Rica. Ex coordinadora del Programa de Investigación en Epistemología Genética y Educación del IIMEC, actualmente el Instituto de investigación en Educación (INIE) de la Universidad de Costa Rica (UCR). Ex Directora y docente de la División de Educación Básica del Centro de Investigación y Docencia en Educación de la Universidad Nacional, Costa Rica. Investigadora del Centro de Investigación y Docencia en Educación de la Universidad Nacional, Costa Rica. Tiene publicaciones en el campo de la Psicología y la Pedagogía.
} 
constante por aumentar conocimientos y transformar, de manera positiva, la realidad circundante, siempre buscando la comprensión y desarrollo integral del ser humano.

Shulman 1986, (citado por Marquès, 2010) plantea que:

El conocimiento no crece de forma natural e inexorable. Crece por las investigaciones de los estudiosos (empíricos, teóricos, prácticos) y es por tanto una función de los tipos de preguntas formuladas, problemas planteados y cuestiones estructuradas por aquellos que investigan. (I 6)

Concordante con ese planteamiento, se han ido desarrollando los diferentes paradigmas en la investigación, así como la diversidad de enfoques que de ellos se derivan. Cualquier investigación en el campo educativo suele estar permeada de buenas intenciones y del deseo de los investigadores y las investigadoras de brindar un aporte a dicho campo, ya sea para conocer un fenómeno, para profundizar en temáticas anteriormente abordadas, o también, para buscar cambios y transformaciones específicas o sociales, a partir de los conocimientos que estudios previos hayan aportado. Independientemente del objetivo de estas, todas buscan la comprensión, profundización o transformación de aspectos en el campo educativo. En esa perspectiva de búsqueda, los diseños mixtos pueden constituirse en un aporte para dicho objetivo.

Como parte de una experiencia personal de investigación en la que la autora de este artículo aplicó un diseño de método mixto para la comprensión de una temática educativa en el ámbito universitario, surge la inquietud de compartir con la comunidad académica y estudiantil, unas reflexiones acerca de dichos diseños y su pertinencia en el abordaje de temáticas de investigación en el campo educativo.

Los diseños mixtos han ido cobrando fuerza día a día y cada vez son más aplicados en investigaciones en diversos campos, en especial, dentro de las Ciencias Sociales, por lo que plantear una revisión sobre los mismos es pertinente para buscar fortalecer su aplicación en el área educativa.

Se organiza el presente artículo, partiendo de algunos planteamientos teóricos acerca de los diseños mixtos, para enmarcar posteriormente, la ejemplificación del diseño utilizado en una investigación que asumió el uso de un diseño con método mixto.

\section{Los diseños de método y modelo mixto}

Durante los años 1960 a 1970, sin otorgarles el nombre de diseños mixtos, se propusieron una serie de estudios e investigaciones en los cuales se procedió a mezclar los enfoques cualitativos y cuantitativos, en áreas del conocimiento como la medicina criminalística. Ruiz (s. f.) menciona que fue Sieber (1973) quien sugirió, también, la mezcla de estudios de caso con encuestas, creando así un nuevo estilo de investigación. Agrega el autor que, por otra parte, Jick, en 1979, introdujo los términos básicos de los diseños mixtos, al recurrir a técnicas e instrumentos proporcionados por paradigmas positivistas y naturalistas para la recolección de datos, dando un lugar prioritario a la triangulación de datos.

En los años 80, se fortaleció ese tipo de opción metodológica y los planteamientos en investigación continuaron combinando los enfoques cuantitativo y cualitativo. También, en esos años, surgió el debate sobre la legitimidad de la investigación mixta y, como corolario, se amplió el concepto de triangulación llevándola más allá de la comparación entre cualitativo y cuantitativo, de modo que aparecieron diversos tipos de triangulación, entre los que es posible mencionar: teorías, 
métodos e investigadores. Pero, también, se diversificaron los enfoques que se proponían dentro del paradigma naturalista (Ruiz, s. f.).

Agrega el autor que Christ (2007) argumenta que la investigación mediante métodos mixtos se ha fortalecido en los últimos veinte años, y los estudios exploratorios cualitativos, seguidos de estudios confirmatorios, han sido comunes y concurrentes. En esa misma línea, Dellinger y Leech (2007) analizan, también, la validez de los métodos mixtos en la investigación.

Tal como lo señalan los autores, durante los años 90, las investigaciones con diseños mixtos se hicieron muy útiles en campos como: Educación, Enfermería, Medicina, Psicología y Comunicación, en el entendido de que el uso de más de un método potenciaba la posibilidad de comprensión de los fenómenos en estudio, especialmente, si estos se refieren a campos complejos en donde está involucrado el ser humano y su diversidad.

Denzin y Lincoln (2002) plantean una revisión profunda acerca de los procesos de triangulación, lo cual aportó a la comunidad científica, en especial en el ámbito cualitativo, importantes elementos que también impactaron de manera positiva las propuestas de investigación denominadas como mixtas.

Hernández, Fernández y Baptista (2003) señalan que los diseños mixtos:

(...) representan el más alto grado de integración o combinación entre los enfoques cualitativo y cuantitativo. Ambos se entremezclan o combinan en todo el proceso de investigación, o, al menos, en la mayoría de sus etapas (...) agrega complejidad al diseño de estudio; pero contempla todas las ventajas de cada uno de los enfoques. (p. 21)

En la misma línea de pensamiento, desde la óptica propuesta por Di Silvestre (s. f.), la complementariedad metodológica ha permeado la comunidad científica por lo que:

(...) se ha ido posicionando en la actualidad una estrategia de investigación que permite combinar la metodología cualitativa y la cuantitativa aún cuando éstas en el pasado se han encontrado en posturas opuestas. Esta estrategia de investigación es la denominada "multimétodos", "métodos mixtos", o "triangulación metodológica", cualquiera sea su nombre ella apunta a la combinación de la metodología cualitativa y la cuantitativa (p. 71)

Para Cameron (2009), la investigación con métodos mixtos ha ido generando cambios metodológicos entre los investigadores y académicos en una variedad de áreas disciplinarias. Creswell y Plano (2007), citados por Cameron (2009), definieron cuatro etapas en la evolución histórica de las investigaciones mixtas, a saber: período formativo (años de los 50 a los 80), período del debate paradigmático (de los años 70 a los 90 tardíos), período del desarrollo procedimental (de los año 80 tardíos al 2000), y la advocacy como un periodo separado del diseño (del 2000 en adelante).

Las investigaciones con complementación metodológica de enfoques cualitativos y cuantitativos fueron evolucionando hasta llegar a conceptualizarlas en función de diseños denominados como de modelo y método mixto.

Para Driessnack, Sousa y Costa (2007): “(...) los métodos mixtos se refieren a un único estudio que utiliza estrategias múltiples o mixtas para responder a las preguntas de investigación y/o comprobar hipótesis" (I 3).

Los estudios propuestos fueron fortaleciendo esas aproximaciones investigativas y dieron origen a planteamientos teóricos interesantes, acerca de su validez y de los procedimientos de triangulación. Desde esa perspectiva, Driessnack et al. (2007) señalan que la triangulación "Se 
refiere a la convergencia o corroboración de los datos recolectados e interpretados a respecto del mismo fenómeno" (p. 4), donde el método de recolección y o interpretación de los datos, bien podría ser diferente.

Es importante señalar que dichos autores retoman la revisión de los diseños de método mixto, en función de su referencial teórico, ya sea cuantitativo o cualitativo y, en ese sentido, señalan que los diseños se pueden clasificar de la siguiente forma:

$\begin{array}{lll}\text { CUAL } & + & \text { cual } \\ \text { CUAL } & \rightarrow & \text { cual } \\ \text { CUAL } & + & \text { cuan } \\ \text { CUAL } & \rightarrow & \text { cuan } \\ \text { CUAN } & + & \text { cuan } \\ \text { CUAN } & \rightarrow & \text { cuan } \\ \text { CUAN } & + & \text { cual } \\ \text { CUAN } & \rightarrow & \text { cual }\end{array}$

La simbología utilizada, ha de interpretarse de la siguiente manera:

+ : Significa que el método secundario se está utilizando simultánea o concomitantemente en el mismo período de recolección de datos.

$\rightarrow$ : Indica que el método secundario se utilizó posterior a la recolección de los datos primarios.

Mayúscula: señala el método o enfoque que tiene priorización en el diseño.

Cual: se refiere al enfoque o métodos cualitativos.

Cuan: se refiere al enfoque o métodos cuantitativos.

Por otra parte, Johnson y Onwuegbuzie (2004) definieron los diseños mixtos como "(...) el tipo de estudio donde el investigador mezcla o combina técnicas de investigación, métodos, enfoques, conceptos o lenguaje cuantitativo o cualitativo en un solo estudio" (p. 17).

Los conocimientos desarrollados, a lo largo del tiempo, permitieron señalar las diferentes características y modalidades que, dependiendo del objeto de estudio, podrían adoptar los diseños mixtos y, en ese sentido, autores como Tashakkori y Teddlie (2003) denominaron los diseños mixtos como el tercer movimiento metodológico, y Mertens (2007) plantea que el enfoque mixto está basado en el paradigma pragmático. Aspecto que también es señalado por Rocco, Bliss, Gallagher y Pérez-Prado (2003), quienes argumentan que los diseños mixtos se fundamentaron en la posición pragmática (el significado, valor o veracidad de una expresión se determina por las experiencias o las consecuencias prácticas que tiene en el mundo) o en la posición dialéctica (hay una mejor comprensión del fenómeno cuando se combinan los paradigmas) y conformaron, así, una tercera fuerza en la investigación. Por otra parte, Moscoloni (2005) hace referencia, al uso de la triangulación en los diseños mixtos, como un elemento de peso para considerarlos como una valiosa alternativa para acercarse al conocimiento de diversos objetos de estudio. 
Los autores y autoras en mención señalan que los diseños mixtos permiten, a las investigadoras y a los investigadores, combinar paradigmas, para optar por mejores oportunidades de acercarse a importantes problemáticas de investigación. En ese sentido, señalan que la investigación mixta se fortaleció, al poder incorporar datos como imágenes, narraciones o verbalizaciones de los actores, que de una u otra manera, ofrecían mayor sentido a los datos numéricos.

Igualmente afirman que los diseños mixtos permiten la obtención de una mejor evidencia y comprensión de los fenómenos y, por ello, facilitan el fortalecimiento de los conocimientos teóricos y prácticos. Destacan, también, que los investigadores han de contar con conocimientos apropiados acerca de los paradigmas que van a integrar mediante los diseños mixtos, de modo que se garantice dicha estrategia.

Señala Cameron (2009) que las investigaciones con diseños mixtos han cobrado fuerza en áreas tan diversas como: consejería, ciencias sociales y humanas, negocios, investigación evaluativa, medicina familiar, lo cual "(...) provee evidencia empírica, de la extensión y utilización de los métodos mixtos en la investigación contemporánea” (traducción personal, p. 4).

Por otra parte, Johnson y Onwuegbuzie (2004), Onwuegbuzie y Leech (2006) plantearon que las investigaciones con un diseño mixto podían ser de dos tipos:

Con modelo mixto: en el cual se combinan en una misma etapa o fase de investigación, tanto métodos cuantitativos, como cualitativos.

Con método mixto: en cuyo caso, los métodos cuantitativos se utilizan en una etapa o fase de la investigación y los cualitativos en otra.

También propusieron una organización de los diseños mixtos en función del paradigma que enfatizaban y del orden o secuencia en el que se aplicaban, en esa línea, plantearon lo siguiente:

Igualdad en el estatus: se da simultaneidad en la aplicación de los métodos y ninguno de ellos se prioriza sobre el otro, solo varía el orden en cuanto a concurrencia o secuencialidad. En el sentido expuesto, dichos diseños pueden expresarse de la siguiente manera:

Concurrente: CUAL + CUAN

Secuencial: CUAL $\rightarrow$ CUAN

CUAN $\rightarrow$ CUAL

Estatus dominante: en esta categoría se ubican los diseños en concordancia con los objetivos de la investigación e interés del proponente y, según la priorización de los enfoques, ya sea el cuantitativo o el cualitativo, la aplicación puede ser tanto secuencial como concurrente.

Concurrente: CUAL $\rightarrow$ cuan

CUAN $\rightarrow$ cual

Secuencial: CUAL $\rightarrow$ cuan

Cual $\rightarrow$ CUAN

$\mathrm{CUAN} \rightarrow$ cual

Cuan $\rightarrow$ CUAL 
Por otra parte, Rocco et al. (2003) plantearon una clasificación acerca de los diseños de método mixto y modelo mixto, en el que señalaron diferentes combinaciones, según el carácter exploratorio o confirmatorio, por lo que vale la pena revisar a continuación dicha organización:

Tipos de diseño con método mixto: Para la clasificación de diseños con método mixto, es decir, aquellos que combinan metodologías cuantitativas y cualitativas, los autores señalados proponen los siguientes tipos:

Tipo I: Investigación confirmatoria, con datos cualitativos y análisis estadístico.

Tipo II: Investigación confirmatoria, con datos cualitativos y análisis cualitativo.

Tipo III: Investigación exploratoria, con datos cuantitativos y análisis estadísticos.

Tipo IV: Investigación exploratoria, con datos cualitativos y análisis estadístico.

Tipo V: Investigación confirmatoria, con datos cuantitativos y análisis cualitativo.

Tipo VI: Investigación exploratoria, con datos cuantitativos y análisis cualitativo.

Tipos de diseño con modelo mixto: Para los diseños que responden a un modelo mixto, en el cual se mezclan estrategias cuantitativas y cualitativas, propusieron los siguientes tipos:

Tipo VII: Simultáneo, investigación confirmatoria o exploratoria. Datos cuantitativos y cualitativos con análisis cualitativos y cuantitativos.

Tipo VIII: Secuencial, por etapas. Una etapa un enfoque, la siguiente el otro. Cada etapa fortalece la anterior.

En la misma línea de pensamiento, Creswell (2008) argumenta que la investigación mixta permite integrar, en un mismo estudio, metodologías cuantitativas y cualitativas, con el propósito de que exista mayor comprensión acerca del objeto de estudio. Aspecto que, en el caso de los diseños mixtos, puede ser una fuente de explicación a su surgimiento y al reiterado uso en ciencias que tienen relación directa con los comportamientos sociales.

Se retoma a continuación la organización de diseños mixtos planteada por Creswell (2008), quien señala que es posible organizarlos de la siguiente manera:

Estrategia secuencial explicatoria: Los resultados cualitativos los utiliza para explicar resultados cuantitativos, el orden es cuantitativo $\rightarrow$ cualitativo, el énfasis es explicar e interpretar relaciones.

Estrategia secuencial exploratoria: Los resultados cuantitativos los usa para explicar los cualitativos, el orden es cualitativo con análisis, seguido de cuantitativo con análisis, el énfasis es explorar un fenómeno.

Estrategia secuencial transformativa: Busca comprender un mejor fenómeno y darle voz a diferentes perspectivas. Utiliza alguna perspectiva teórica y el énfasis está en la transformación.

Estrategia concurrente de triangulación: En un mismo estudio busca confirmar, correlacionar o corroborar. Utiliza alguna perspectiva teórica, en la interpretación busca la integración. Se recopilan datos cuantitativos y cualitativos simultáneamente.

Estrategia concurrente de nido: Estudia diferentes grupos o niveles para obtener una perspectiva más amplia. Simultáneamente, se recogen datos cuantitativos y cualitativos. Se integra en el análisis.

Estrategia concurrente transformativa: Utiliza alguna perspectiva teórica, recoge datos cuantitativos y cualitativos simultáneamente. Se integra en el análisis.

Como se ha señalado anteriormente, son variadas las clasificaciones que se aportan en la bibliografía acerca de los diseños mixtos. Es importante advertir que algunas de esas propuestas son 
novedosas y buscan amparar las investigaciones formuladas dentro de diseños mixtos, y consideran no sólo la integración metodológica, sino también el orden, etapas o secuencias que las caracterizan, así como los tipos de diseño (Voils, Sandelowski, Barroso y Heselblad, 2008).

En una reciente investigación, realizada por la autora de este ensayo, acerca de las vivencias universitarias y caracterizaciones de docentes universitarios y el clima de aula, desde la visión de estudiantes de la Universidad Nacional, para acercarse a este objeto de estudio del campo educativo (Pereira, 2010), se hizo uso de un diseño de método mixto, por lo que se recurre a dicha experiencia para ejemplificar, a continuación, una posible aplicación de los diseños mixtos en el campo pedagógico.

\section{Una experiencia concreta}

\section{El objeto de estudio:}

Los planteamientos teóricos sobre la temática de las características de docentes señalan una tendencia a abordar cuestiones referidas a las mismas, desde la visión de los formadores, de las instituciones, de las especialidades, así como desde las estrategias didácticas, materiales y recursos.

De igual manera, se han destacado las características de docentes desde la visión del manejo del contenido, pero hay pocas investigaciones, que apunten a la perspectiva estudiantil como fuente de datos, para identificar sus características deseables, aunque, cabe aclarar, ha habido participación estudiantil en aspectos referidos a la evaluación de cursos. Por lo tanto, la investigación mencionada respondió a la necesidad de acercarse a los rasgos del personal docente, desde una óptica menos conocida, como lo es la mirada estudiantil.

Es claro el planteamiento en las últimas épocas, acerca de que el estudiantado es el centro del proceso de aprendizaje; no obstante, por lo que se reporta en los estudios, son escasas las ocasiones en las cuales es este grupo el que brinda su opinión acerca de sus docentes, excepto en cuanto a evaluación del desempeño en determinados cursos.

En especial, a raíz de los procesos de acreditación, las instituciones educativas han otorgado al alumnado la oportunidad de evaluar, no solo los cursos recibidos, sino también a las profesoras y profesores que los impartieron. Cabe destacar que con frecuencia dichas evaluaciones enfatizan, prioritariamente, en aspectos referidos al manejo del contenido por parte de cuerpo docente, a los recursos didácticos y estrategias de aula, más que a las dinámicas interactivas, emociones y clima. En la línea señalada, la investigación desarrollada apostó a recuperar la perspectiva estudiantil acerca de las dinámicas interactivas, la caracterización del personal docente universitario y como parte de ello, el clima del aula.

La investigación se desarrolló en el ámbito de la División de Educación Básica (DEB), Unidad Académica de la Universidad Nacional encargada de la formación de docentes.

Como parte del proceso de negociación, se establecieron los primeros contactos mediante los cuales se obtuvo la anuencia de la Unidad Académica para respaldar el trabajo, debido al interés en la temática que planteaba la investigación.

El permiso para ingresar a la institución se otorgó por parte de las autoridades universitarias. De igual manera, se negoció con el grupo de docentes de la DEB, el permiso para ingresar a las aulas y solicitar la anuencia de los estudiantes y las estudiantes del nivel de licenciatura de las carreras con énfasis en Preescolar, I y II ciclos y Educación Especial (Integración) para la aplicación de instrumentos. 
Se incorporan tres macro categorías de análisis, a saber:

- Dinámicas interactivas profesor/profesora-alumno/alumna y dinámicas interactivas alumnosalumnas entre sí.

- Características del docente y la docente universitaria

- Clima de aula

La investigación se propuso indagar cuáles eran las características reales y deseables del docente y la docente universitaria, identificadas por el estudiantado de la División de Educación Básica de la Universidad Nacional. Así seconstituyó un posible perfil para el personal docente universitario, quien promueve no solo el desarrollo del estudiante y de la estudiante en su ámbito personal, sino también en el cognoscitivo. De igual manera, buscó la comprensión de dichas dinámicas y su repercusión en el clima de aula.

\section{La aproximación metodológica:}

La investigación realizada cuyo título es: "La mirada de estudiantes de la universidad nacional hacia el docente y la docente: sus características y clima de aula" (Pereira, 2010) se enmarcó dentro de un diseño de método mixto (Ver figura 1), compartió lo planteado por Ruiz (s. f.) cuando afirma que la investigación mixta busca obtener un conocimiento más amplio y profundo acerca del objeto de estudio y comprenderlo integralmente.

En cuanto a las clasificaciones sobre diseños de método o modelo mixtos, planteados en la bibliografía, la investigación realizada, respondió a los siguientes criterios:

- Diseño de método mixto, con status dominante y de orden secuencial cuan $\rightarrow$ CUAL (Johnson y Onwuegbuzie, 2004).

- En cuanto al tipo de estrategia seguida, la investigación respondió a una estrategia secuencial explicatoria, con una secuencia cuantitativa-CUALITATIVA, con integración de datos en la interpretación y cuyo énfasis fue explicar e interpretar relaciones (Creswell, 2003).

En el sentido expuesto, la investigación realizada se enmarcó prioritariamente dentro de un paradigma naturalista, con un enfoque cualitativo que buscó una posible caracterización del docente y la docente, dentro de las dinámicas interactivas en el aula universitaria, y el clima de aula, desde la perspectiva estudiantil. Dentro de los planteamientos que refuerzan este abordaje pueden destacarse los siguientes:

Desde la perspectiva de Taylor y Bogdan (citados por Rodríguez, Gómez y Gil, 1996), se concibe la investigación cualitativa como “(...) aquella que produce datos descriptivos: las propias palabras de las personas, habladas o escritas y la conducta observable" (p. 33).

En esa misma línea de pensamiento señala Cea (2001) que “(...) el paradigma cualitativo se asocia a la epistemología interpretativa (dimensión intersubjetiva), centrada en el sujeto individual y en el descubrimiento del significado, los motivos y las intenciones de su acción” (p. 46).

Por ello, Salgado (2007) afirma que el diseño, en el marco de una investigación predominantemente cualitativa, es flexible y abierto, razón por la cual ha de ajustarse a las características, no solo del objeto de estudio, sino también a las condiciones en que se circunscribe. 
La investigación realizada y que ejemplifica el diseño, otorgó un lugar predominante al enfoque cualitativo, dada la necesidad de profundizar en las dinámicas relacionales (docentes y alumnos /alumnas y alumnos y alumnas entre sí), en el clima de aula y en un posible perfil docente universitario, a partir de la perspectiva estudiantil.

Por tanto, la investigadora compartió el criterio de Mertens (2007), quien argumenta que una tarea fundamental del investigador que busca la comprensión de un mundo complejo consiste recurrir al análisis de la experiencia vivencial desde la visión de quienes la experimentan, como una manera para "(...) comprender sus diversas construcciones sociales sobre el significado de los hechos y el conocimiento" (p. 8).

Señalan Hernández et al. (2003) que para el análisis de datos en el enfoque mixto se sugiere “(...) incluir una sección donde se presente el método, la recolección y el análisis de datos tanto cuantitativos como cualitativos" (p. 634). En cuanto a los resultados de los estudios con diseño mixto, dichos autores sugieren que los mismos se presenten con el esquema de la triangulación, se busque consistencia entre los resultados de ambos enfoques y se identifiquen posibles contradicciones o paradojas.

El diseño mixto (DIMIX) utilizado contempló dos fases, las que, por cronología en la aplicación, se han definido como fase I y fase II. La fase I respondió a un enfoque cuantitativo, el cual, temporalmente, se desarrolló de primero. La segunda, dentro de un enfoque cualitativo, el que, para su diseño y desarrollo, se nutrió de la fase I. Cada una de estas fases se describe a continuación.

\section{Fase I: Enfoque cuantitativo, método descriptivo}

La fase I conllevó la utilización del método descriptivo. Hernández et al. (2003), citando a Danke, afirman que los estudios descriptivos “(...) buscan especificar las propiedades, las características y los perfiles importantes de personas, grupos comunidades o cualquier otro fenómeno que es sometido a un análisis" (p. 117).

Por otra parte, Babbie (2000) plantea que "Uno de los principales objetivos de muchos estudios sociales científicos es describir situaciones y acontecimientos (...) estas descripciones suelen ser más fieles y precisas que las casuales" (p. 74). Desde esa misma óptica, Hernández et al. (2003) indican que algunos de ellos describen situaciones, eventos o fenómenos y que se busca detallar lo que se investiga.

Con la premisa de que el estudiantado universitario tiene mucho que aportar en la comprensión de las dinámicas relacionales y de que las experiencias de formación universitaria experimentadas, les faculta para ofrecer opiniones válidas, acerca de las características deseables en el personal docente universitario, se planteó, en la fase I, el acercamiento a la temática.

Para ello, se empleó un cuestionario para recopilar los rasgos del personal docente universitario desde la perspectiva estudiantil. Lo cual permitió elaborar un mapeo del objeto de estudio, con un mayor número de estudiantes y elaborar un posible perfil, de las características deseables del personal docente universitario, así como de las características reales de los docentes y las docentes que les han formado y del impacto en el clima de aula. 


\section{Fase II: Enfoque cualitativo, método fenomenológico}

La fase II asumió el método fenomenológico. Este, por su naturaleza introspectiva, favorece la búsqueda y comprensión de características del docente y la docente, desde la perspectiva estudiantil. Dicho método recupera las características esenciales de las experiencias y la esencia de lo que se experimenta. Se aplicó la técnica del conversatorio y se recurrió, además, a una prueba de oraciones incompletas para fortalecer la comprensión de la temática abordada.

El término fenomenología viene de la Filosofía, y hay autores que sugieren la utilización del concepto interpretativismo para referirse a este y otros métodos, ya que enfatizan en la construcción social de la realidad, en los aspectos cognitivos, afectivos y contextuales, que permiten el conocimiento de un objeto de estudio. El método fenomenológico puede considerarse como eminentemente participativo, dado que el significado solo puede ser creado a través de la interacción (LeCompte y Schensul, 1999).

Por el tipo de datos que se buscaron y con el acercamiento a las comprensiones de los participantes, la investigación generó teorías sustantivas, aprehensibles por el método fenomenológico que permitieron ahondar en la comprensión de las dinámicas interactivas en el ámbito universitario, desde la perspectiva estudiantil y sus vivencias, al retomar la realidad interna y personal del estudiantado participante.

Se compartió la idea de que hay una fuerte relación entre el sujeto y el objeto de conocimiento y de que, en esa construcción, intervienen, no solo las capacidades cognoscitivas, sino también las afectivas en las interacciones con los otros, de ahí la pertinencia de asumir la perspectiva fenomenológica.

Para el proceso de reducción del dato se recurrió a la codificación, identificación de palabras claves y segmentos, identificación de patrones recurrentes, categorización. Mediante matrices se desplegó la información para facilitar la identificación de tendencias, patrones, contradicciones, ausencias, que permitieran la conceptualización, para posteriormente, abocarse a su validación por triangulación

Las estrategias de validación de la información dieron inicio con la formalización del análisis de los instrumentos mediante aplicación piloto y criterio de jueces.

De igual manera, la aplicación estuvo a cargo de la investigadora, así como la tabulación y el correspondiente análisis, de modo que la interacción de investigadores se vio controlada mediante este procedimiento unilateral.

Para la temática propuesta, fue conveniente realizar el análisis de los datos mediante procedimientos que garantizaran su validez, por lo que se efectuó:

- Contrastación de los datos obtenidos, con el aporte de estudios previos en el campo de investigación.

- Triangulación por teoría, resultados del método descriptivo y del fenomenológico, informantes y fuentes de recolección de datos. La triangulación fue valiosa para identificar las características ideales del personal docente universitario y sus repercusiones en sanas dinámicas interactivas en el ámbito universitario.

En la siguiente figura se esquematiza, mediante un diseño de método mixto (DIMIX), el procedimiento metodológico seguido en la investigación titulada: "La mirada de estudiantes de la Universidad Nacional hacia el docente y la docente: sus características y clima de aula" (Pereira, 2010). 

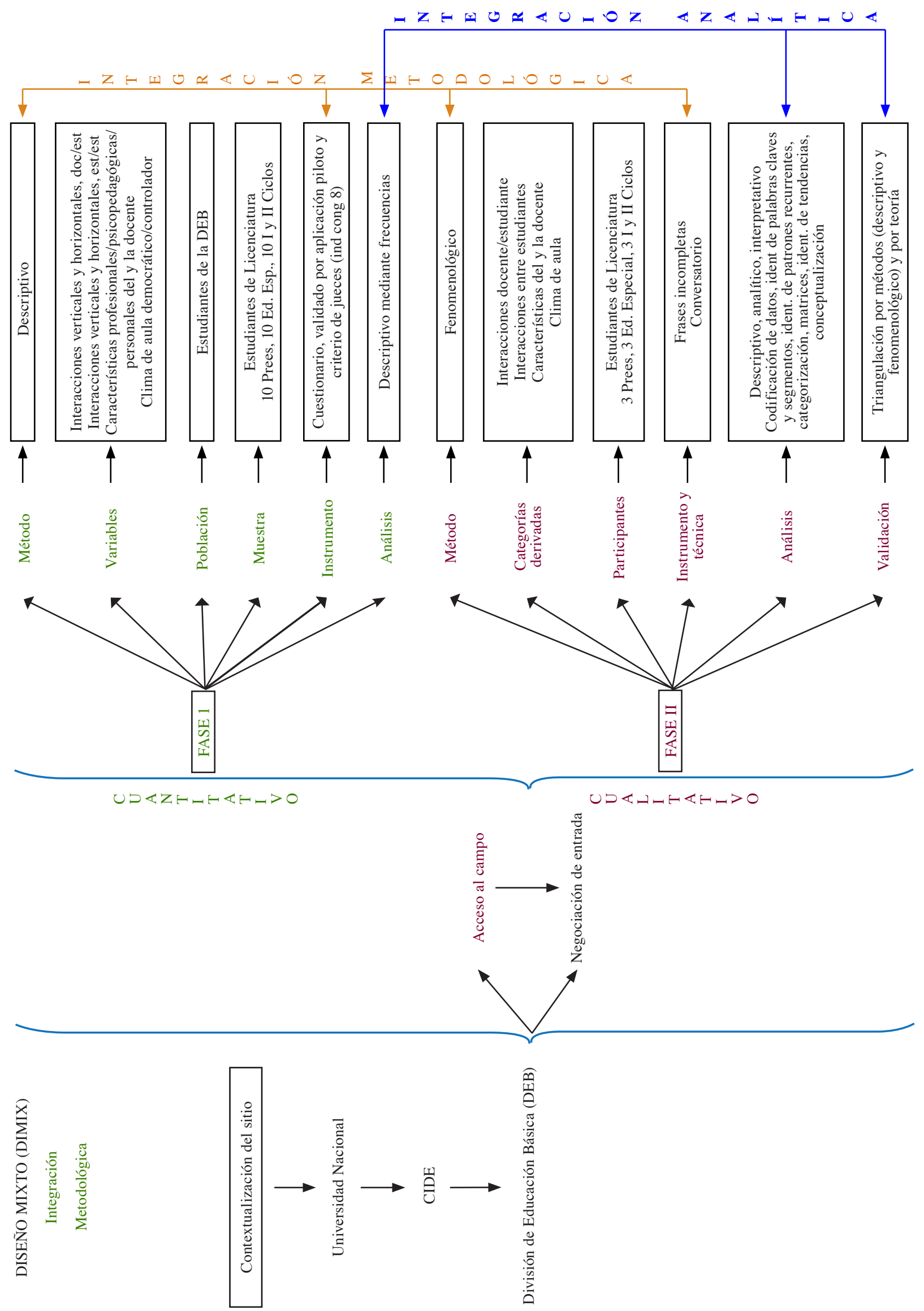


\section{Integración de datos para análisis:}

El primer manejo de la información evidenció la necesidad de optar por un análisis más integrado, buscando la comprensión de las dinámicas del aula universitaria tal como lo plantea la recomendación teórica (Dellinger y Leech, 2007; Driessnack et al. (2007). En procura de dicha integración, los datos en ambas fases fueron transcritos textualmente, se recurrió al cromado y a la ubicación de los datos, en función de categorías analíticas. Cuando se hizo uso de los criterios expresados por las estudiantes, se respetó la forma, contenido y escritura, tal cual fueron expresados por ellas.

Los datos recopilados fueron nuevamente analizados por las estudiantes participantes, mediante un conversatorio con el propósito de respetar su visión de la temática de estudio y reforzar los puntos que fueran necesarios. La información se presentó, de manera integrada, cuando hubo semejanzas entre los aportes dados por las estudiantes de los diferentes énfasis de carrera y, separadamente, cuando existieron divergencias.

Los datos se organizaron por participante, por énfasis de carrera, por instrumento y de manera consistente se mantuvo el orden por participante. En el caso específico de las transcripciones de los datos de la prueba de oraciones incompletas, en las tablas se mantuvo el orden por participante, de modo que las respuestas 1-4 son de estudiantes de Educación Preescolar, las 5-7 corresponden a estudiantes de I y II Ciclos y las 8-10 son de Educación Especial.

En la presentación de los datos se ofrece información general acerca de las estudiantes participantes y posteriormente, se exponen los datos en forma integrada, retomando aquellos de carácter cuantitativo mediante el uso de gráficos y tablas. Los datos de naturaleza cualitativa se presentaron en tablas y mediante la transcripción de frases expresadas por las participantes. La integración de datos cuantitativos y cualitativos se dio en función de categorías de análisis que permitieron un mejor acercamiento al objeto de estudio. Cabe mencionar que la bibliografía recomienda que, para la búsqueda de dicha integración, se aplique la técnica de triangulación, por lo cual en la investigación realizada constituyó una excelente opción para el análisis e integración, y permitió el logro de la profundización temática mediante dicha técnica apoyada en la organización por categorías analíticas.

Lo dicho no es un procedimiento sencillo, ya que requiere el manejo de los datos desde los enfoques utilizados, buscando la integración coherente, por lo que la triangulación se constituye en un valioso aliado para lograrlo. Con lo planteado, el deseo es animar al lector y lectora a acercarse al recurso de los diseños de método y modelo mixto para la realización de investigaciones en educación.

\section{Consideraciones finales:}

Como puede observarse, es viable el planteamiento de un diseño de método mixto para la aproximación a temáticas de estudio en el ámbito pedagógico, en especial, cuando hay una evidente intención del investigador o investigadora para otorgar voz a los participantes y a las participantes; en ese sentido, cuando no solo se desea la obtención de datos numéricos, sino también se busca la visión más íntima del participante, los datos cualitativos cobran un papel relevante, tal como fue el caso con el que se ejemplifica este ensayo.

La experiencia que se relató es un ejemplo preciso de cómo quien investiga puede plantear un acercamiento a la temática de estudio mediante la aplicación de métodos o modelos mixtos, los cuales le otorgan la posibilidad de poder profundizar y comprender de mejor manera el fenómeno o 
situación estudiada. En este caso particular, priorizando la mirada de los sujetos participantes como un elemento clave para la construcción de conocimiento en un área específica, como fue el ámbito universitario, lo que permitió caracterizar al personal docente y el clima de aula.

Es importante resaltar, tal como se señaló anteriormente, que el investigador y la investigadora ha de hacer un esfuerzo para la integración de datos en busca de la unidad que le permitirá una mejor comprensión de su tema de estudio. En este proceso, resulta sumamente valioso el recurso de la triangulación. En el caso particular de la experiencia desarrollada, la misma enfatizó en la teoría, informantes, métodos y técnicas e instrumentos de recolección de datos.

Es igualmente válido destacar que existen muchas y variadas formas de acercarse a una temática de estudio, las cuales van en concordancia con la posición epistemológica de los investigadores y las investigadoras, los recursos de que disponga, el esfuerzo por la recuperación de datos cuantitativos o cualitativos, los fines de la investigación, el interés por adquirir nuevos conocimientos, profundizar en conocimientos ya construidos o promover cambios como parte de los procesos investigativos, entre otras razones. No obstante lo dicho, el presente ensayo contextualiza una experiencia concreta, mediante el uso de diseños de método mixto, que se considera puede ser de utilidad para la comprensión de temáticas educativas.

\section{Referencias bibliográficas}

Babbie, E. (2000). Fundamentos de la investigación social. México, D. F.: Internacional Thompson Editores.

Cameron, R. (2009). The use of mixed methods in VET research. [El uso de métodos mixtos en la investigación en educación vocacional]. Recuperado de http://www.avetra.org.au/ papers-2009/papers/12.00.pdf

Cea, M. Á. (2001). Metodología cuantitativa. Estrategias y técnicas de investigación social. ( $3^{\mathrm{a}}$ Reimpresión). Madrid, España: Editorial Síntesis.

Christ, T. (2007, julio). A Recursive Approach to Mixed Methods Research in a Longitudinal Study of Postsecondary Education Disability Support Services [Un enfoque recursivo para Métodos de investigación mixta en un estudio longitudinal de los servicios de apoyo a la discapacidad en educación post secundaria]. Journal of Mixed Methods Research, 1(3), 226-241. doi: $10.1177 / 1558689807301101$

Creswell, J. (2003). Outline: Creswell's Research Design [Esquema: Diseño de investigación de Creswell]. Recuperado de http://www.ics.uci.edu/alspaugh/human/crewel.html

Creswell, J. (2008, febrero). Mixed Methods Research: State of the Art. [Power Point Presentation]. University of Michigan. Recuperado de sitemaker.umich.edu/creswell.workshop/files/ creswell lecture slides.ppt

Dellinger, A. y Leech, N. (2007, octubre). Toward a Unified Validation Framework in Mixed Methods Research [Hacia un marco de validación unificado en métodos de investigación Mixtos]. Journal of Mixed Methods Research, 1(4), 309-332. doi: 10.1177/1558689807306147 
Denzin, N. y Lincoln, Y. [Eds.]. (2002, noviembre). The Qualitative Inquiry Reader [El lector de investigación cualitativa]. Forum: Qualitative Social Research, 3(4), Art. 35. Recuperado de http://www.qualitative-research.net/index.php/fqs/article/viewArticle/780/1692

Di Silvestre, C. (s.f.). Metodología cuantitativa versus metodología cualitativa y los diseños de investigación mixtos: conceptos fundamentales. Argentina: ANACEM. Recuperado de http://173.255.237.28/anacem.cl/public/wordpress/wp-content/uploads/2009/03/metodologiacuantitativa-versus-cualitativa.pdf

Driessnack, M., Sousa, V. y Costa, I. (setiembre-octubre, 2007). Revisión de los diseños de investigación relevantes para la enfermería: parte 3: métodos mixtos y múltiples. Revista LatinoAmericana de Enfermagem, 15(5), 179-182. Recuperado de http://www.scielo.br/pdf/rlae/ v15n5/es v15n5a24.pdf

Hernández, R., Fernández, C. y Baptista, P. (2003). Metodología de la investigación (3ª ed.). México: Editorial Mc Graw-Hill.

Johnson, B. y Onwuegbuzie, A. (2004, October). Mixed Methods Research: A Research Paradigm Whose Time Has Come [Los métodos de investigación mixtos: un paradigma de investigación cuyo tiempo ha llegado]. Educational Researcher, 33(7), 14-26. Recuperado de http://edr. sagepub.com/cgi/content/abstract/33/7/14

LeCompte, M. y Schensul, J. (1999). Designing and Conducting Ethographic Research [Diseño y realización de investigación etnográfica]. London: AltaMira Press.

Marquès, P. (2010). Ciencia y metodologías de investigación. Diseño de una investigación educativa. Recuperado de http://peremarques.pangea.org/edusoft.htm

Mertens, D. (2007). Transformative Paradigm Mixed Methods and Social Justice [Paradigma transformativo metodos mixtos y justicia social]. Journal of Mixed Methods Research, 1(3), 212225. doi: $10.1177 / 1558689807302811$

Moscoloni, N. (2005). Complementación metodológica para el análisis de datos cuantitativos y cualitativos en evaluación educativa. Revista Electrónica de Metodología aplicada, 10(2), 1-10. Recuperado de http://www.psico.uniovi.es/rema/v10n2/moscoloni.pdf

Onwuegbuzie A. J. y Leech, N. L. (2006, septiembre). Linking Research Questions to Mixed Methods Data Analysis Procedures. Qual Report; 11(3), 474-498. Recuperado de http://www. nova.edu/ssss/QR/QR11-3/onwuegbuzie.pdf

Pereira, Z. (2010). La mirada de estudiantes de la Universidad Nacional hacia el docente y la docente: sus características y clima de aula. (Tesis doctoral sin publicar). Universidad Estatal a Distancia. San José, Costa Rica.

Rocco, T., Bliss, L., Gallagher, S. y Pérez-Prado, A. (2003, Spring). Taking the Next Step: Mixed Methods Research in Organizacional Systems [Tomando el siguiente paso: 
Métodos mixtos de investigación en sistemas organizacionales]. Information Technology, Learning, and Performance Journal, 21(1), 19-29. Recuperado de http://www.osra.org/itlpj/ roccoblissgallagherperez-pradospring2003.pdf

Rodríguez, G., Gómez, J. y Gil, J. (1996). Métodos de la investigación cualitativa. Málaga, España: Ediciones ALJIBE.

Ruiz, C. (s. f.) Enfoque cualitativo, cuantitativo y mixto. Recuperado de http://www.scribd.com/ $\underline{\text { doc/2726742/ }}$

Salgado, A. (setiembre, 2007). Investigación cualitativa: diseños, evaluación del rigor metodológico y retos. LIBERABIT, 13, 71-78. Recuperado de www.scielo.org.pe/pdf/liber/v13n13/a09v13n13. pdf

Tashakkori, A. y Teddlie, C. [Eds.]. (2003). Handbook of Mixed Methods in Social \& Behavioral Research [Manual de métodos mixtos en investigación social y del comportamiento]. Thousand Oaks: Sage Publications. Recuperado de http://books.google.co.cr/books?hl=es\&l $\underline{\mathrm{r}=\& \mathrm{id}=\mathrm{F} 8 \mathrm{BFOM} 8 \mathrm{DCKoC} \& \text { oi=fnd \&pg=PR9\&dq=Handbook }+ \text { of }+ \text { mixed }+ \text { methods }+ \text { in }+ \text { social }}$ $+\% 26+$ behavioral+research\&ots $=\mathrm{gSiQBBqzNk \& sig=nFDTw4B3zKb}-\mathrm{kP56vBzwZEEvC8}-$ $\mathrm{v}=$ onepage $\& \mathrm{q} \& \mathrm{f}=$ false

Voils, C., Sandelowski, M., Barroso, J. y Hsselblad, V. (2008, February). Making Sense of Qualitative and Quantitative Findings in Mixed Research Síntesis Studies [Analizando hallazgos cuantitativos y cualitativos en síntesis de estudios con diseños mixtos]. Field Methods, 20(1), 3-25. doi: $10.1177 / 1525822 X 07307463$ 